\title{
In-situ Spectroscopy of Catalysts
}

\author{
Bert M. Weckhuysen \\ Department of Inorganic Chemistry and Catalysis, Debye Institute, \\ Utrecht University, Sorbonnelaan 16, 3508 TB Utrecht, The Netherlands
}

\section{CONTENTS}

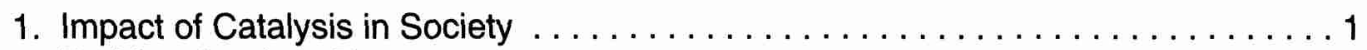

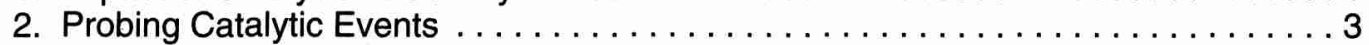

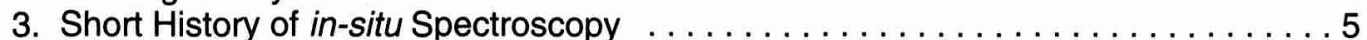

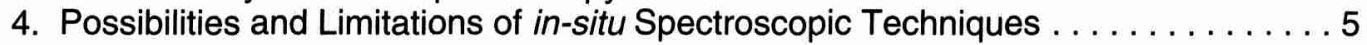

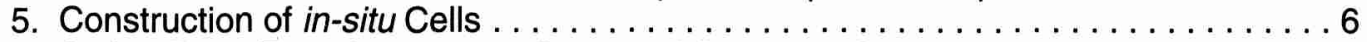

6. Active Sites, Reaction Intermediates and Spectator Species . . . . . . . . . . . 8

7. Potential Roadmap for in-situ Spectroscopic Studies $\ldots \ldots \ldots \ldots \ldots \ldots \ldots \ldots$

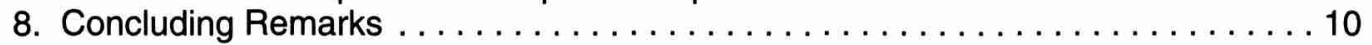

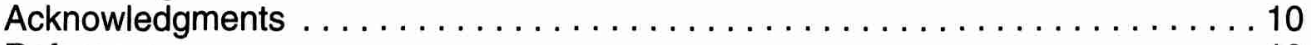

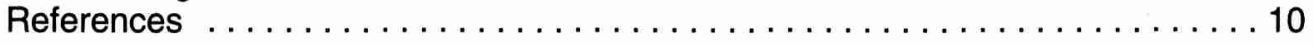

\section{IMPACT OF CATALYSIS IN SOCIETY}

A catalyst facilitates a chemical reaction by lowering the activation energy $\left(E_{a}\right)$ of the reaction pathway [1-3]. This is schematically shown in Figure 1 for a condensation reaction between substrate molecules $\mathrm{A}$ and $\mathrm{B}$. The condensation product $\mathrm{C}$ is formed from a transition state structure $\mathrm{C}^{\prime}$ or $\mathrm{C}^{\prime \prime}$ in case of the non-catalyzed or catalyzed reaction, respectively. Catalysts increase the reaction rate and therefore the efficiency of the overall chemical process by lowering the activation energy $\left(E_{a}{ }^{\prime \prime}<E_{a}{ }^{\prime}\right)$. Besides being responsible for a higher activity, catalysts also direct a reaction that influences the selectivity of a chemical process. It is especially the latter property which makes catalysis the key-technology for the development of a sustainable society.

Car exhaust systems are surely one of the most recognized examples of catalyst technology by non-chemists and these catalysts represent an important market for catalyst manufacturers. On the other hand, people should be aware that almost any chemical product we use in daily life has been in contact with a catalyst. In this respect, it is no wonder that more than $85 \%$ of all bulk and fine chemicals are produced with the aid of one or more catalysts $[4,5]$. As an example, Figure 2 shows a picture of an industrial plant for the production of olefins, a building block for making e.g. plastics. Each of the five reactors of this installation is loaded with more than 40 tons of a $\mathrm{Cr} / \mathrm{Al}_{2} \mathrm{O}_{3}$ dehydrogenation catalyst. The importance of catalyst materials is further underlined by the wide variety of industrial catalytic processes as illustrated in Table 1 $[4,5]$. Examples are the production of ammonia, methanol, plastics and pharmaceuticals. They are also used to remove sulfur from crude oil and methane to make e.g. low-sulfur fuels.

Figure 3 gives an overview of most of the catalytic materials presently available. Three types of catalytic materials can be distinguished: biocatalysts, homogeneous catalysts and heterogeneous catalysts [6]. The former group includes $e . g$. enzymes and cells. They are considered ideal catalysts since they usually operate in aqueous solutions at room temperature, normal pressure and with nearly $100 \%$ selectivity, making them the ideal catalysts for oxidation reactions. They also serve as an inspiration source for people designing the ligand core of metals and transition metal ions in homogeneous catalysis. Heterogeneous catalysts-the main materials covered in this textbook-are solids. They can be either bulk or supported materials, several types and compositions of which are available. Supports can be either amorphous (for example, Pt clusters can be loaded on a porous alumina as shown in Figure 4) or crystalline (for example, zeolite ZSM-5 crystals 


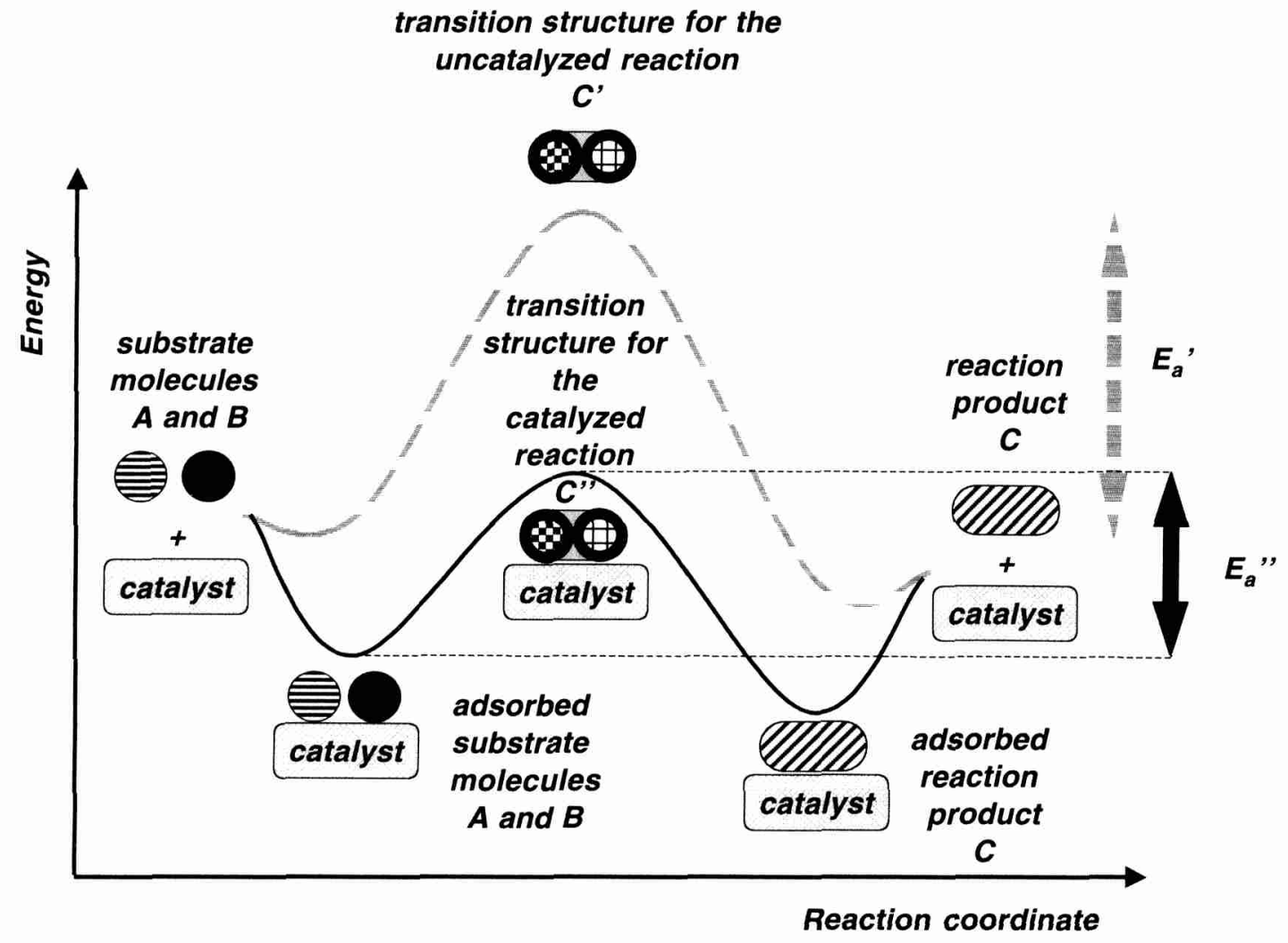

Figure 1. Energy scheme for a catalyzed and uncatalyzed reaction between two substrate molecules $A$ and $B$ leading to the formation of reaction product $\mathrm{C}$. The reaction between $\mathrm{A}$ and $\mathrm{B}$ leads to the formation of a transition structure $\mathrm{C}^{\prime \prime}$ or $\mathrm{C}^{\prime}$ with different activation energies $E_{a}{ }^{\prime \prime}$ or $E_{a}{ }^{\prime}$ in the presence and absence of a catalyst material.

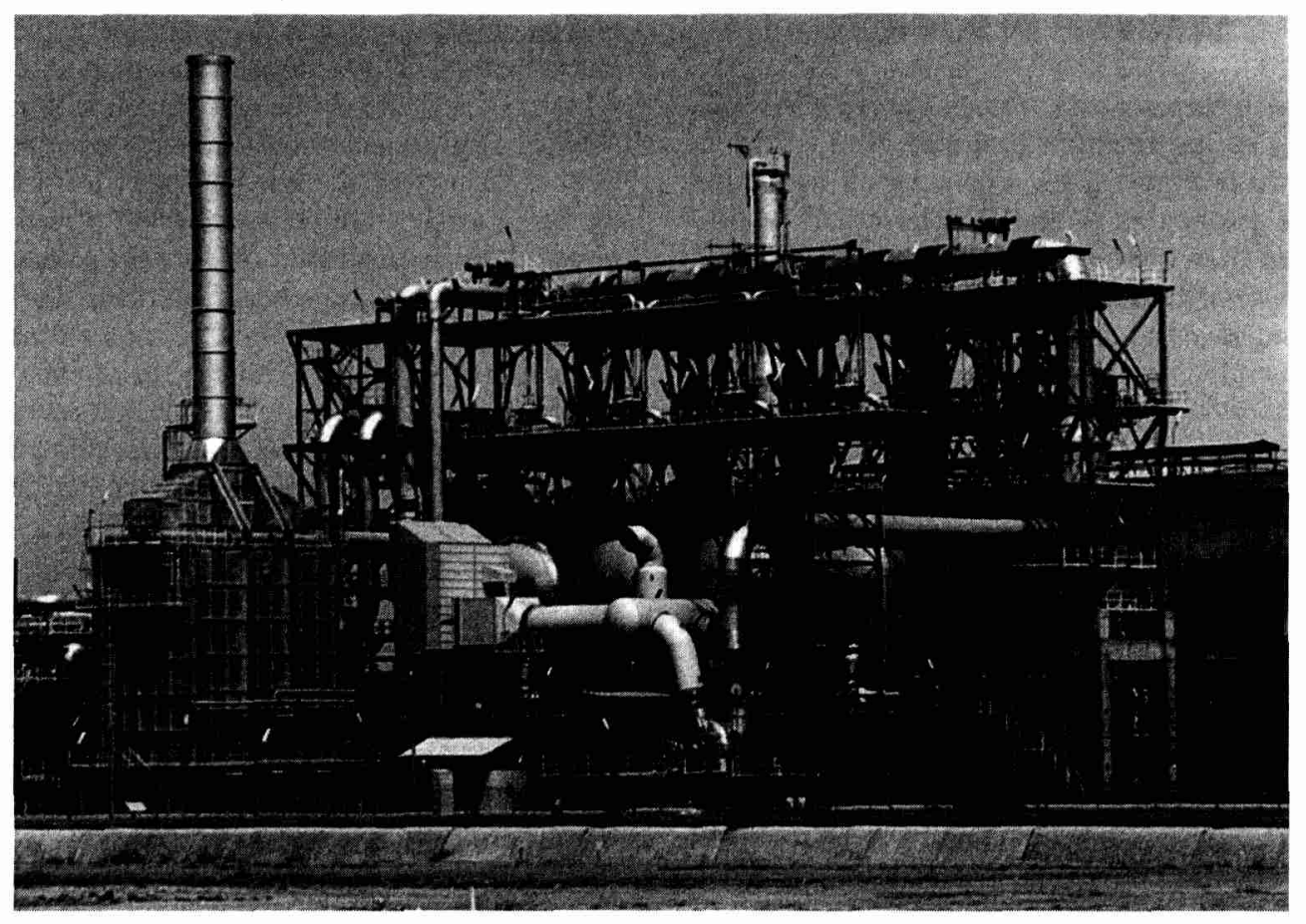

Figure 2. Plant consisting of five fixed-bed catalytic reactors used for the industrial production of olefins, such as propylene. The catalyst is a chromium oxide supported on a porous aluminium oxide. Each catalyst bed has a height of approximately $1-1.5 \mathrm{~m}$. 
Table 1. Important industrial processes making use of catalyst materials.

\begin{tabular}{ll}
\hline \multicolumn{1}{c}{ Catalyst } & \multicolumn{1}{c}{ Industrial applications } \\
\hline $\mathrm{V}_{2} \mathrm{O}_{5} / \mathrm{TiO}_{2}$ & Selective reduction of $\mathrm{NO}_{\mathrm{x}}$ with $\mathrm{NH}_{3}$ \\
$\mathrm{CrO}_{3} / \mathrm{Al}_{2} \mathrm{O}_{3}$ & Dehydrogenation of light alkanes \\
$\mathrm{CrO}_{3} / \mathrm{SiO}_{2}$ & Polymerization of olefins \\
$\mathrm{Re}_{2} \mathrm{O}_{7} / \mathrm{Al}_{2} \mathrm{O}_{3}$ & Metathesis of olefins \\
$\mathrm{Ni} / \mathrm{Al}_{2} \mathrm{O}_{3}$ & Steam reforming of methane \\
$\mathrm{CuO} / \mathrm{ZnO} / \mathrm{Cr}_{2} \mathrm{O}_{3}$ & Synthesis of methanol \\
$\mathrm{Pt} / \mathrm{Al}_{2} \mathrm{O}_{3}$ & Isomerization of light gasoline \\
$\mathrm{K} 2 \mathrm{O} / \mathrm{Cr}_{2} \mathrm{O}_{3} / \mathrm{Fe}_{3} \mathrm{O}_{4}$ & Dehydrogenation of ethylbenzene to toluene \\
$\mathrm{Zeolite} \mathrm{Y}$ & Fluid catalytic cracking of crude oil \\
$\mathrm{Co} / \mathrm{TiO}_{2}$ & Fischer-Tropsch synthesis \\
$\mathrm{Fe} / \mathrm{K}_{2} \mathrm{O} / \mathrm{Al}_{2} \mathrm{O}_{3}$ & Ammonia synthesis \\
$\mathrm{Pt} / \mathrm{Rh}_{\mathrm{CoS}} / \mathrm{MoS}_{2} / \mathrm{Al}_{2} \mathrm{O}_{3}$ & Ammonia oxidation \\
\hline & Desulfurization of crude oil \\
\hline
\end{tabular}

as shown in Figure 5). Supports mostly possess a high surface area and porosity. These properties are needed to enhance the number of catalytic active sites as well as to avoid diffusion problems toward these active sites.

\section{PROBING CATALYTIC EVENTS}

Understanding how, for example, two substrate molecules A and $\mathrm{B}$ in Figure 1 are transformed into the condensation product $C$ is a real challenge [7-11]. Ideally, chemists would like to have fundamental insight in the reaction cycle and the manner in which a catalyst material may affect this reaction cycle. This would enable scientists to design better catalysts to increase the conversion of A and B and the selectivity towards the desired reaction product $\mathrm{C}$.

A catalytic cycle is a hypothesized network of reaction steps describing how the transformation of the substrate molecule towards the envisaged reaction product occurs over one or a cascade of catalytic active sites. The development of such a network may occur at different levels of sophistication of which the simplest is the formulation of the elementary reaction steps and a description of the overall nature of the catalytic active site. In contrast, the most sophisticated and desired level is the description of the transformation process in terms of (a) reac-

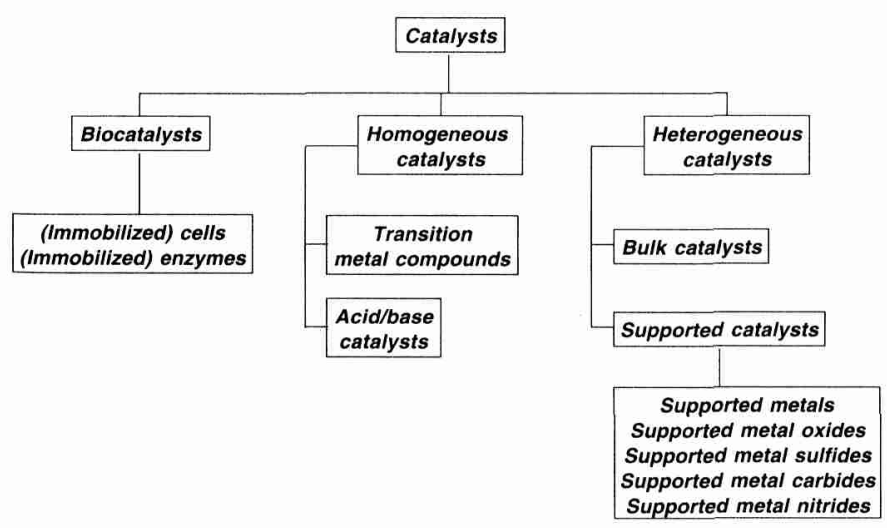

Figure 3. General overview of the field of catalysis, including the domains of homogeneous catalysis, heterogeneous catalysis and biocatalysis.

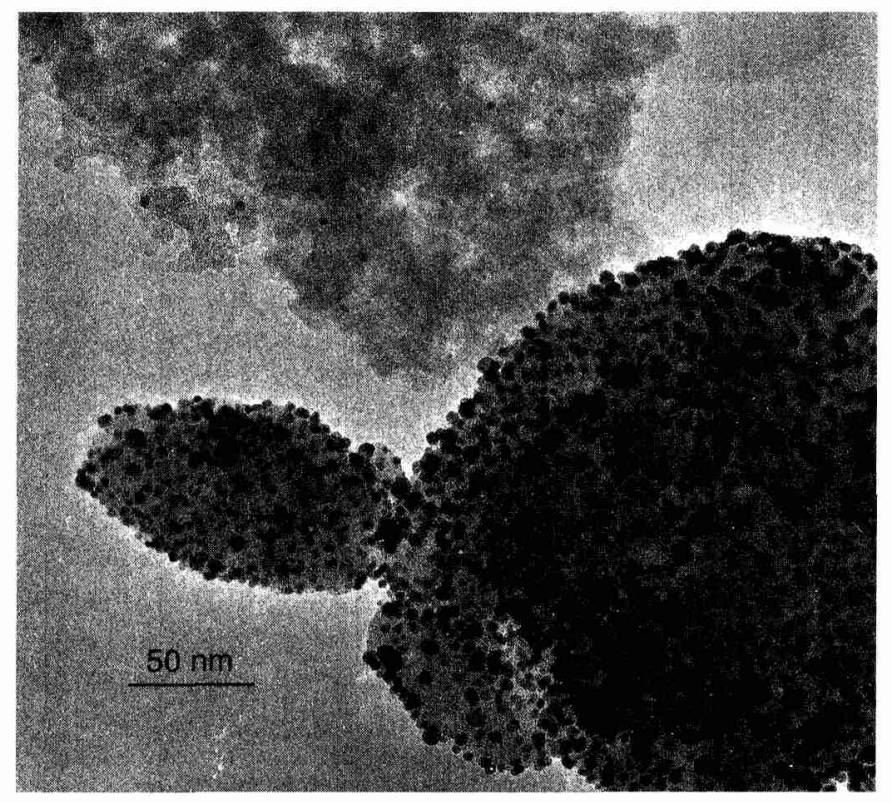

Figure 4. Transmission electron microscopy (TEM) picture of Pt particles supported on a porous alumina support, a catalyst material used of the isomerization of light gasoline.

tion trajectories together with the reaction dynamics and kinetics along those trajectories and (b) molecular insight in catalytic active sites and the dynamics toward their coordination environment. Understanding catalytic reactions at a fundamental level implies that the developed reaction network should be experimentally and theoretically tested in sufficient detail. Although many chemists have been working for decades to de-

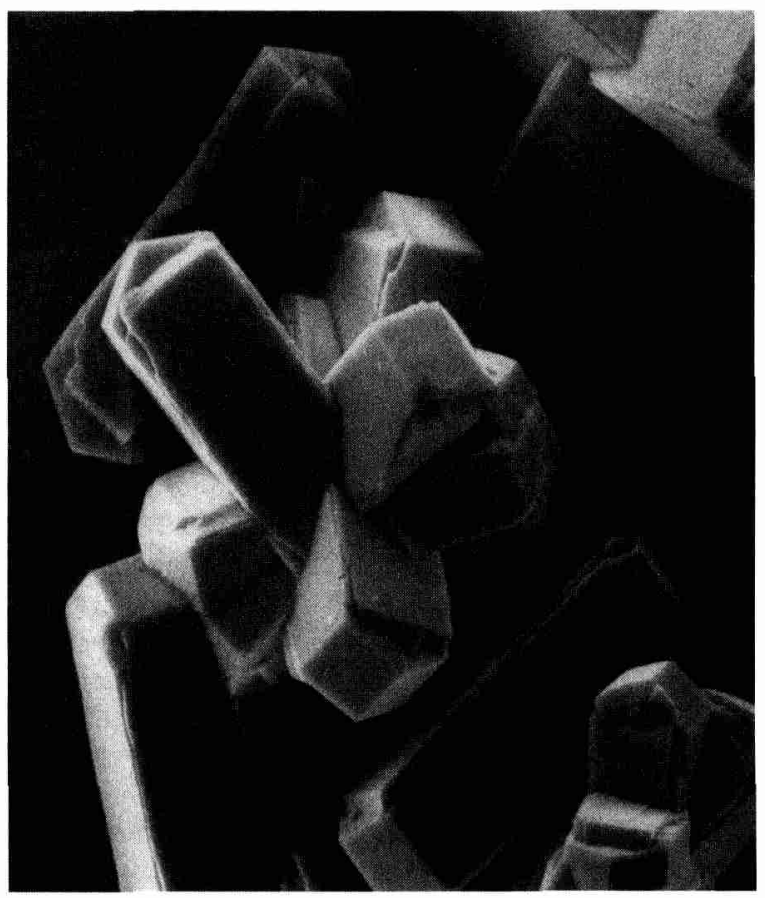

Figure 5. Scanning electron microscopy (SEM) picture of some crystals of zeolite ZSM-5, a catalyst material for methanol-to-olefins (MTO) conversion. 
mystify catalytic events, only in a limited number of cases has such detailed understanding been achieved.

The way to do so is to gather sufficient detailed information about the catalyst material at each step of its lifespan [6]. Figure 6 illustrates the lifespan of a catalyst starting from its genesis up to its deactivation [12]. In a first step, the catalyst material is synthesized and an example of such preparation is the impregnation of a porous solid (S) with an aqueous solution of a transition metal oxide $\left(M O_{x}{ }^{n+}\right)$. The next step is heating the freshly prepared catalyst material in oxygen, often referred to as calcination, which will result in an anchoring of the transition metal oxide onto the surface through interaction with its hydroxyl groups. As a consequence, a $M O_{x}{ }^{n+}-\mathrm{S}$ catalyst precursor material is formed. In a third step, the catalyst precursor is activated and this activation treatment often consists of a reduction treatment in which the oxidation state of the active metal oxide phase is changed $\left(M O_{x}{ }^{p+}-S\right.$ with $\left.p<n\right)$. The obtained solid is now ready for catalytic operation and will be able to convert a substrate molecule to the desired reaction product. Gradually, the catalyst will deactivate because of $e . g$. a change in dispersion of the active metal oxide phase. In other cases, coke is formed deactivating the active sites. In some particular cases, the deactivated catalyst can be regenerated.

Conventional spectroscopy now focuses on the study of the freshly prepared, calcined, reduced or deactivated catalyst material and such measurements are usually done under normal conditions; i.e., at room temperature and under ambient pressure. Although interesting information can already be obtained, the gathered information does not provide any insight about what is really going on in the catalyst material during catalytic reaction. In other words, only knowing the "beforereaction part" and/or "after-reaction part" does not deliver information on how the catalyst behaves in the industrial reactor and what species is active in the catalytic process. As indicated by G. A. Somorjai, "it is like studying a life with access only to the prenatal and postmortem states" [13]. In order to surpass this problem, researchers often measure an active catalyst after quenching the material to room temperature and transferring it in e.g. a glove box to a spectroscopic cell for characterization. This methodology can be coined "fossilization of the catalyst material". Although already much more relevant, this approach is still not ideal because the catalyst material may restructure during cooling down and because of the absence of the reactant molecules.

Therefore, the only solution is to adapt spectroscopic techniques in such a way that they are able to monitor catalysts "in action"; in other words, to track the physicochemical processes taking place in an active catalyst in real time and under operating conditions. This is the field of in-situ spectroscopy $[10,11,14-25]$. On the ohter hand, the name operando spectroscopy is gaining popularity in the field because the term defines in a better way the experimental conditions under which spectroscopic data of a catalyst surface should be measured [24]. Since the name in-situ spectroscopy is still much more widespread, we will stick to this word. Some authors, however, will use in their own book chapters already the name operando spectroscopy. In any case, performing the ideal in-situ spectroscopic experiment is far from easy and may remain a fantasy as suggested by J. F. Haw [25]. According to this author, a perfect in-situ experiment would look inside an industrial reactor and reveal the most intimate details of a surface chemical reaction, including transition states of reactions. The envisaged sensor would profile chemical compositions on the scale of catalyst particles as well as spatial variations over the volume of the reactor with a high time resolution. Haw differentiates, therefore, between the "purest" definition of the term "in-situ spectroscopy", in which the spectroscopic measurement should be made under

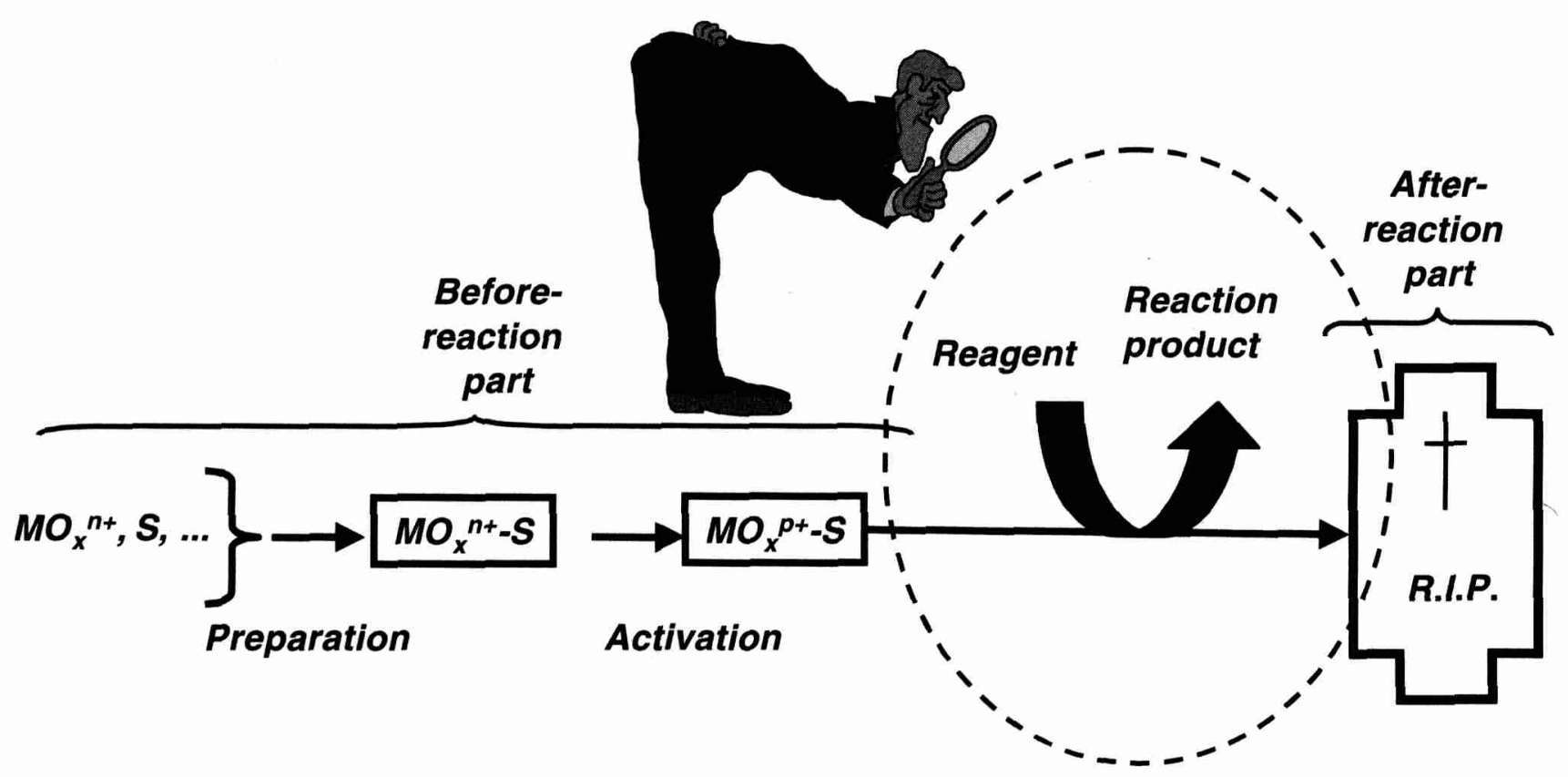

Figure 6. The lifespan of a catalyst, indicating the preparation, activation, catalytic event and deactivation process of the catalyst material. M, $\mathrm{O}$ and $\mathrm{S}$ are the transition metal ion, oxygen and support, respectively. The superscripts refer to the oxidation state of the metal ion. 
reaction conditions and a more inclusive definition, which refers to any spectroscopic result that is relevant to reaction conditions and teaches us about catalytic chemistry. In my opinion, the ultimate dream should still be to perform the ideal in-situ experiment. This should be possible if we can develop technology to such advanced levels that no or only minor concessions in time scale, temperature, pressure and cell design have to be made and that the related data processing and handling becomes less cumbersome.

\section{SHORT HISTORY OF IN-SITU SPECTROSCOPY}

To the best of my knowledge, the history of in-situ spectroscopy in catalysis started as early as 1954 with two seminal papers from the R. P. Eischens group [26, 27]. These authors studied with infrared (IR) spectroscopy the interaction of $\mathrm{CO}$ molecules with $\mathrm{Cu}, \mathrm{Pt}, \mathrm{Pd}$ and $\mathrm{Ni}$ supported on $\mathrm{SiO}_{2}$ and of $\mathrm{NH}_{3}$ molecules with cracking catalysts. It is important to recall that these studies can still not be seen as real in-situ investigations of catalysts. Nevertheless, they represent an important step forward to the in-situ approach since they consider the importance of the dynamics of a catalyst surface in the presence of adsorbates. Indeed, R. P. Eischens was most probably the first to construct a spectroscopic-reaction cell for measuring IR spectra of heterogeneous catalysts. The special cell consisted of two cylindrical $\mathrm{CaF}_{2}$ discs and a Pyrex tube. The catalyst film was deposited on one of the $\mathrm{CaF}_{2}$ windows. The cell was then placed in a furnace with the $\mathrm{CaF}_{2}$ discs loosely in place. Molecules were chemisorbed on the catalyst material after heating and evacuation. The $\mathrm{CaF}_{2}$ windows were then pushed in place and fixed to the Pyrex tube. The spectroscopic cell was transferred to the spectrometer and IR spectra of the catalyst were measured at room temperature. Around the same period, Russian groups also reported the measuring of IR spectra of different molecules on siliceous materials, but these materials cannot yet be considered as catalytic solids [28-30].

Since these initial studies in IR spectroscopy, a continuous progress toward the use of in-situ spectroscopic techniques

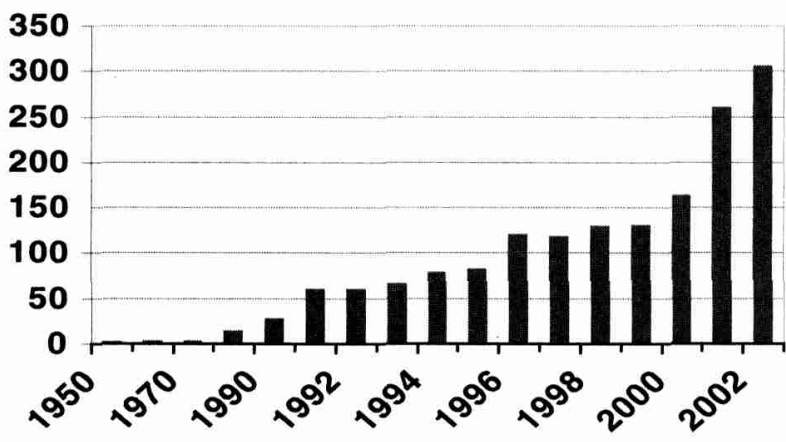

\section{Year}

Figure 7. Estimation of the number of publications on in-situ spectroscopy of catalytic solids during the last five decades. can be observed. This is illustrated in Figure 7, which shows the evolution of the number of in-situ spectroscopy papers in the catalysis literature [31]. It is clear that in the 1960s, 1970s and 1980s, the number of in-situ papers was still relatively low, but starting from the year 1990, the application of in-situ spectroscopic techniques for studying catalytic problems became as important as it is today. M. Jacoby of Chemical \& Engineering News even stated that in-situ spectroscopy is a real buzz-word in catalysis these days [13]. It is no wonder that the number of research papers on in-situ spectroscopy totalled more than 300 in the year 2002.

\section{POSSIBILITIES AND LIMITATIONS OF IN-SITU SPECTROSCOPIC TECHNIQUES}

Several research strategies can be used to investigate the fundamental relations between the physicochemical properties of a catalyst material and its catalytic activity and selectivity [7]. This is schematically illustrated in Table 2 . Scientists are always faced with the dilemma between the level of information they would like to obtain about a catalyst and the relevance of this information regarding the real catalytic event. One approach is to model the catalytic surface with that of a single crystal. Application of appropriate surface science techniques will lead to valuable information on the atomic scale of the catalyst material. The disadvantage, however, is that most of these techniques are carried out in ultra-high or high vacuum, and not under relevant reaction conditions. In this respect, one should be aware that some surface science techniques, such as X-ray photo-electron spectroscopy, are inching into interesting pressure ranges that allow the introduction of reactants at several millibars.

The other approach is to investigate real catalyst systems with techniques under reaction conditions or, as is still often done, under a controlled environment after quenching the chemical reaction to $e$.g. liquid nitrogen temperature. The disadvantage of this approach is that atom-scale information cannot (yet) be obtained and one (still) ends up with an overall picture of the active catalyst material under reaction conditions. Furthermore, in-situ spectroscopy is rather involved since incident photons (ranging from radiowaves to X-rays), charged particles (e.g. electrons) and neutral particles (e.g. neutrons) will probe the gas and/or liquid phase surrounding the catalyst as well as the catalyst material itself. In the case of a catalytic solid, both the (catalytic active) surface and the (catalytic inactive) bulk are probed. All these facts may lead to a skewed interpretation of the spectroscopic data.

One can make a survey of the number of papers on catalytic solids published in 2002 using a particular in-situ characterization technique. The outcome of such literature search is that the most important characterization techniques are infrared spectroscopy (IR) and Raman spectroscopy (RS). These techniques will be covered in detail in part I of the book. Other heavily applied techniques are X-ray absorption spectroscopy (mainly EXAFS and XANES) and more information on these techniques will be presented in part II. Part III of the book deals with in-situ magnetic resonance techniques. Nuclear magnetic resonance (NMR) has been used in the past, however, very interesting results are now obtained with electron paramagnetic resonance (EPR) as well. Part IV 
Table 2. Research strategies available to relate the physicochemical properties of catalysts with their catalytic performances. The in-situ characterization techniques covered in this book are indicated in italics.

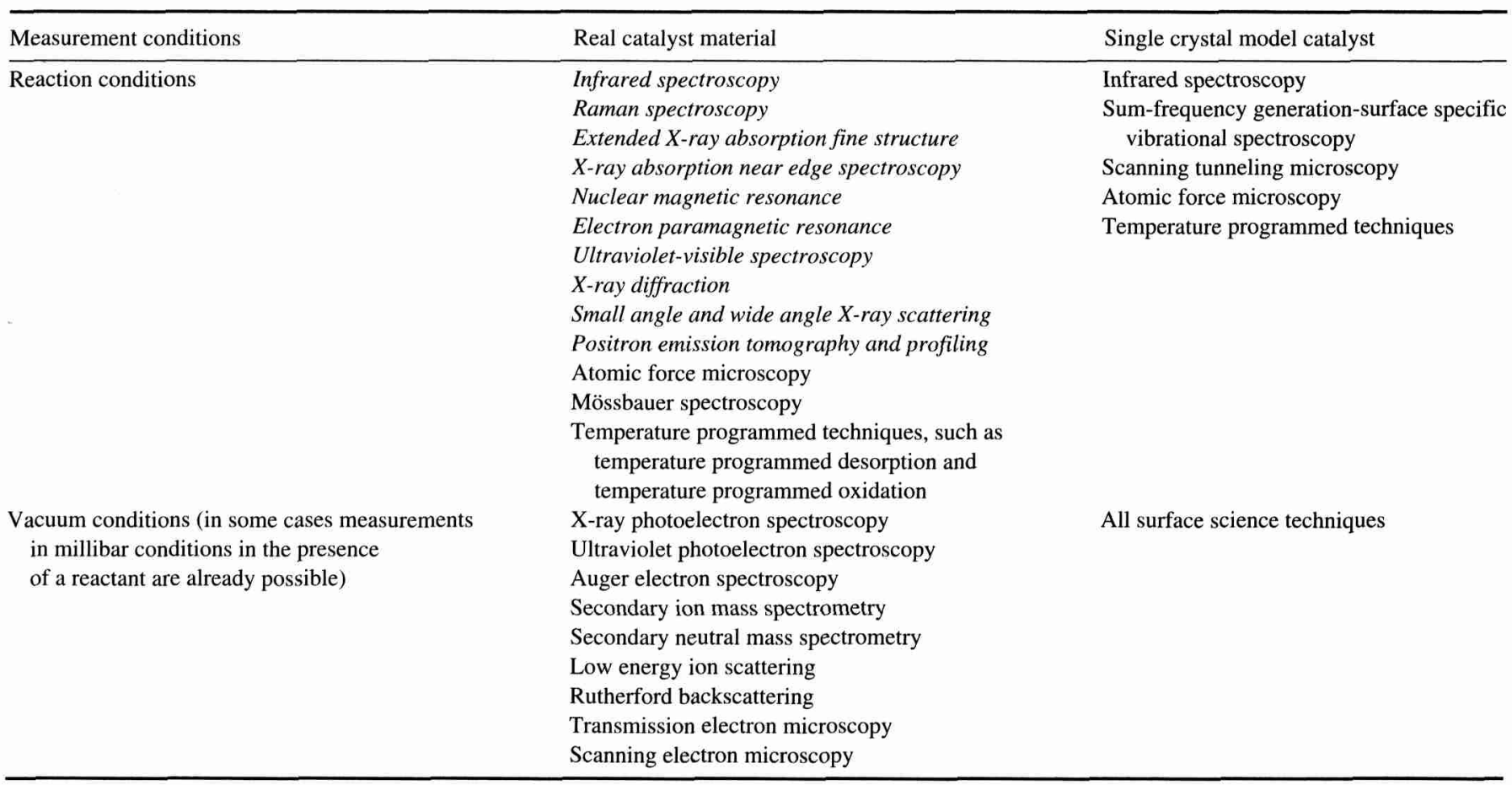

covers less used in-situ techniques such as ultraviolet-visible (UV-VIS) spectroscopy; X-ray diffraction (XRD) and X-ray scattering (SAXS and WAXS); positron emission tomography (PET) and positron emission profiling (PEP).

Each of the presented techniques has its own advantages and disadvantages to reveal details on the active catalyst surface. Table 3 summarizes the potentials and limitations of the spectroscopic techniques covered in the book for conducting in-situ studies on supported metal oxide catalysts. Each technique has a specific potential for quantitatively or qualitatively probing the oxidation state, the coordination environment and the dispersion of a metal ion. Needless to say, none of the characterization techniques listed in Table 3 will be capable of providing all the information needed for complete characterization of the catalytic solid under reaction conditions. In this respect, it is remarkable that most of the in-situ characterization studies found in the literature survey mentioned above are based on one spectroscopic technique only. A possible explanation for this observation is that each spectroscopic technique is associated with specific expertise and thus specialists. Catalysis laboratories, due to a lack of skilled manpower, can therefore rarely have all these in-situ techniques in house and apply them at advanced levels.

\section{CONSTRUCTION OF IN-SITU CELLS}

In order to obtain relevant information about the catalyst material, spectroscopic and catalytic measurements have to be done on the same catalyst at the same time under optimal spectroscopic and catalytic conditions $[10,11]$. The catalyst material has to be placed in a catalytic reactor, which allows the measuring of spectroscopic data at $e . g$. high temperatures and pressures in the presence of a substrate. The catalytic activity and selectivity of the catalyst material can then be obtained via on-line gas chromatography or mass spectrometry. The appropriate design and construction of spectroscopicreaction or in-situ cells is a crucial step in obtaining connected spectroscopic and catalytic data. One challenge in designing in-situ cells is that optimal conditions for performing spectroscopic and catalytic measurements are not identical and thus a compromise has to be found between both types of measurements. Generally speaking, high measurement temperatures and pressures always give rise to less resolved spectra, which make their analysis more difficult. Furthermore, finding a good compromise between spectroscopy and catalysis often implies that sub-optimal catalytic performances are measured in a spectroscopic-reaction cell. Nevertheless, there are now in-situ cells available enabling the study of catalytic processes up to $700-1000^{\circ} \mathrm{C}$ and $50-100$ bars with properties very close to those of real catalytic reactors.

The following aspects have to be considered if one wants to construct a high quality in-situ cell for investigating catalysts in action [10]:

1. Catalyst temperature: It is not uncommon that the actual temperature at the catalyst bed in the cell is lower or higher than indicated by a temperature read-out. This phenomenon is responsible for lower or higher catalytic performances as would be expected for a real catalytic reactor. These changes in temperatures will also affect the spectroscopic data, a problem that can be partially solved by careful choice of thermocouple placement e.g. near the catalyst bed, and a homogeneous heating system surrounding the optical parts of the cell. Inferior 
Table 3. Spectroscopic techniques covered in the textbook and their potentials and limitations to obtain information about supported metal oxide catalysts under working conditions.

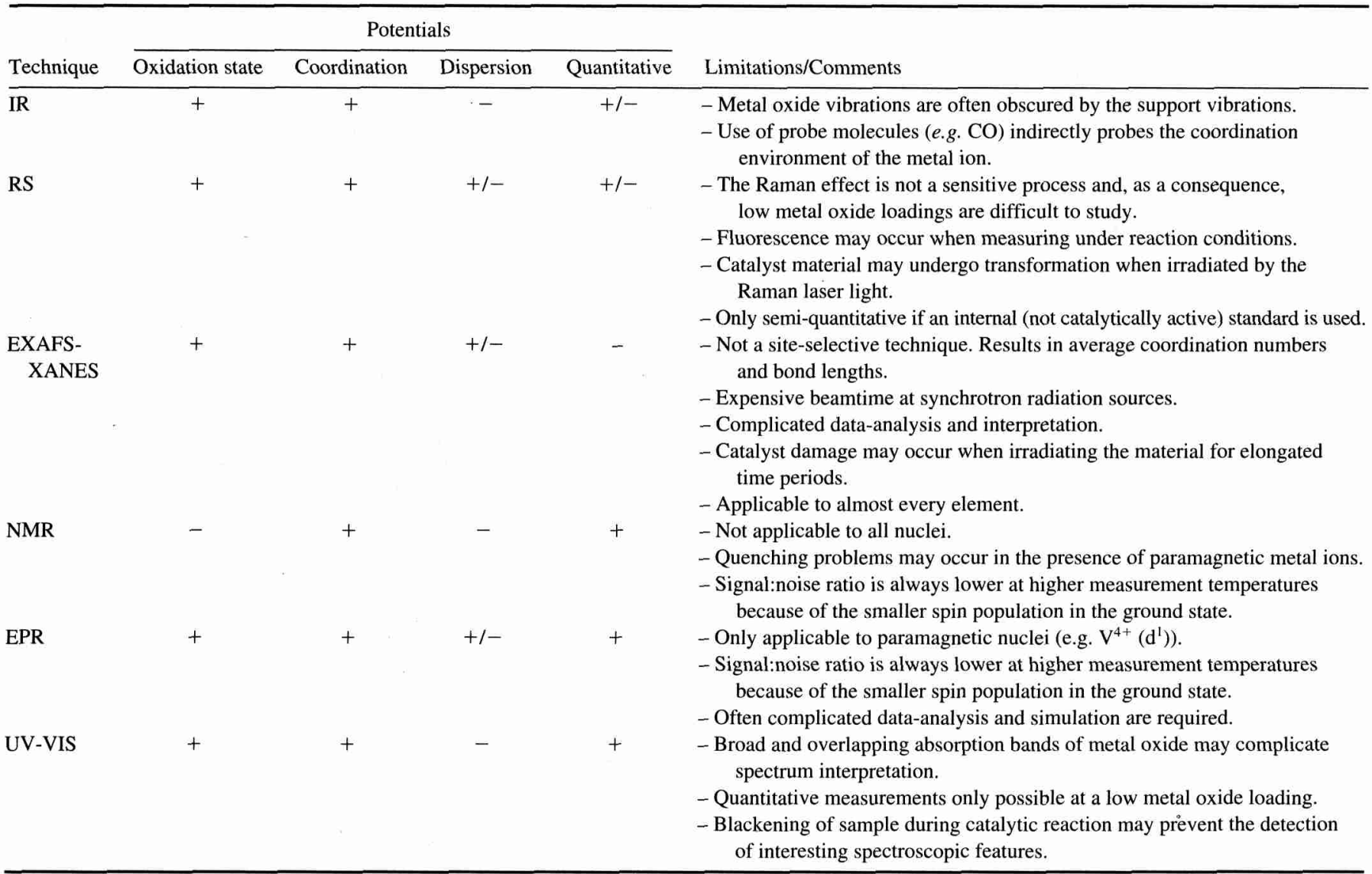

catalytic activity is also partially due to some heat loss via the spectroscopic window. This may result in temperature gradients over the catalyst material, which in turn will affect the spectroscopic and catalytic data. A temperature increase of the catalyst bed can also be related with the light source used for measuring spectroscopic data. Previous experience has demonstrated that the use of a Raman laser source easily may increase the catalyst temperature $10^{\circ} \mathrm{C}$ or more.

2. Catalyst form: Catalytic solids can be placed in the in-situ cell either as powders, granules or as pressed wafers. Powdered samples or catalyst granules allow measuring under flowing conditions. Such continuous operation is very similar to a fixed bed catalytic reactor as gases flow through the catalyst bed. In the case of catalyst wafers, the reactant gases can only flow along the sides of the pressed wafer and irreproducible gas stream profiles and concentration gradients may occur. This may result in inferior catalytic properties and spurious spectroscopic data.

3. Catalyst damage: This is an underestimated problem. The used light source for in-situ spectroscopy can affect the physicochemical properties of the catalyst material. An example is the laser-induced dehydration of catalysts when applying in-situ Raman spectroscopy. The intense laser source results in an increase of the temperature of the catalyst near the laser focus spot. It is re- ported that UV Raman laser radiation may even lead to coke formation during catalyst investigations. This problem can be solved by lowering the measurement time, lowering the laser power, changing the laser frequency, using a rotating laser beam or by using an in-situ cell with a rotating sample. Catalyst damage can also take place with synchrotron-based radiation (e.g. EXAFS-XANES), however careful selection of the beam-line station and measurement time can (partially) solve this problem.

4. Catalyst activity and selectivity: Aside from the intrinsic lower activity and selectivity, one might also expect another activity/selectivity pattern in the in-situ cell. This can be due to some background activity of the in-situ cell itself (due to the presence of $e . g$. a thermocouple, metal parts, etc.). Another problem is that gas molecules, such as oxygen and water, may leak into the in-situ cell, giving rise to different reaction pathways. An example of the latter could be that a total combustion process, instead of a selective oxidation reaction, is studied by in-situ spectroscopy. Alternatively, reagents and reaction products may also leak out of the in-situ cell. Therefore, on-line monitoring of the reaction products formed in the in-situ cell is crucial.

5. Reaction cell volume: The dead space volume of an in-situ cell should be as small as possible in order to reduce the absorption of $e . g$. the gas phase, which could 
interfere with the absorption of the active catalyst material, as well as to avoid gaseous bypass in the cell. A small reaction cell volume is also beneficial if one wants to perform transient reaction studies.

6. Spectroscopic window: High-quality spectroscopic windows (e.g. high-purity quartz for UV-VIS measurements) must be used due to the already inherent lower signal-to-noise (S:N) ratio of the in-situ spectra. This is also important in the case of in-situ EPR measurements because small impurities of $\mathrm{Fe}^{3+}$ within the in-situ cell material may easily show up in the spectroscopic data. Sometimes problems may occur by focusing the light source on the catalyst material (e.g. Raman laser), giving rise to signals of the spectroscopic window.

7. Safety: This is especially true for spectroscopic experiments at high pressures and reaction temperatures. A safety valve could be installed in order to avoid the explosion of the in-situ cell or the blow out of the spectroscopic window (the weakest part of the set-up). Appropriate temperature isolation and a separate hood are critical when an in-situ cell is designed for high temperature applications.

\section{ACTIVE SITES, REACTION INTERMEDIATES AND SPECTATOR SPECIES}

Solid catalyst materials - the main topic of this textbookare very complex materials as illustrated in Figure 8. Several species can be present at the catalyst surface simultaneously. The following species can be distinguished:

1. Active sites; i.e., surface species, which can catalyze a chemical reaction. They are denoted as $A_{1}, A_{2}$ and $A_{3}$. Each of these species may bind a substrate, giving rise to an active site-reaction intermediate surface complex (e.g. $\left.A_{3}-R_{3}\right)$. Reaction intermediates $\left(R_{1}, R_{2}\right.$ and $\left.R_{3}\right)$ are mostly short-lived and therefore difficult to detect. The different active sites may exhibit a different activity and selectivity pattern toward substrate molecules and a particular active site may be responsible for an unwanted side reaction as well, resulting in some by-product formation. It is important to stress that the active sites are mostly present in low concentrations and identification is often difficult.

2. Spectator species; i.e., surface species, which do not actively participate in the catalytic reaction, but are formed during the reaction and thus are in an appreciable amount at the surface, complicate the spectroscopic analysis.

It is clear that it will be necessary to discriminate between true active sites and spectator species, however this is only possible through the careful selection of a battery of complementary characterization techniques and the development of site-selective spectroscopic techniques with a sufficient discriminative power. Another challenge is the quantification of active sites in a working catalyst. The ideal situation would be a quantitative determination of each fraction of surface species on the basis of spectroscopic data only, but to the best of my knowledge, this has not been achieved to date due to the lack of data regarding the extinction coefficients of mostly unusual surface species formed in an active catalyst. The extinction coefficients of such species cannot be simply found in spectroscopic textbooks nor can they be measured independently in a specific experiment. Equally important to consider is the fact that the surface species formed in a working catalyst are mostly unusual identities, and consequently, the interpretation of the spectroscopic data in terms of active sites, reaction intermediates and spectator species is far from trivial. A solution can be given by using advanced theoretical tools for calculating experimental spectroscopic data. This requires the generation of theoretical spectra of relevant computer cluster models on a series of potential active sites. A close match between the theoretical and experimental spectra allows a detailed description of the structure of the active site or reaction intermediate. Based on this knowledge, a reaction cycle can be proposed and mechanistic insight can be obtained.

\section{POTENTIAL ROADMAP FOR IN-SITU SPECTROSCOPIC STUDIES}

As stated above, until now, a lot of spectroscopic techniques in the catalysis field have been used under experimental conditions which are still far from relevant catalytic conditions. In cases where realistic catalytic conditions were chosen, the

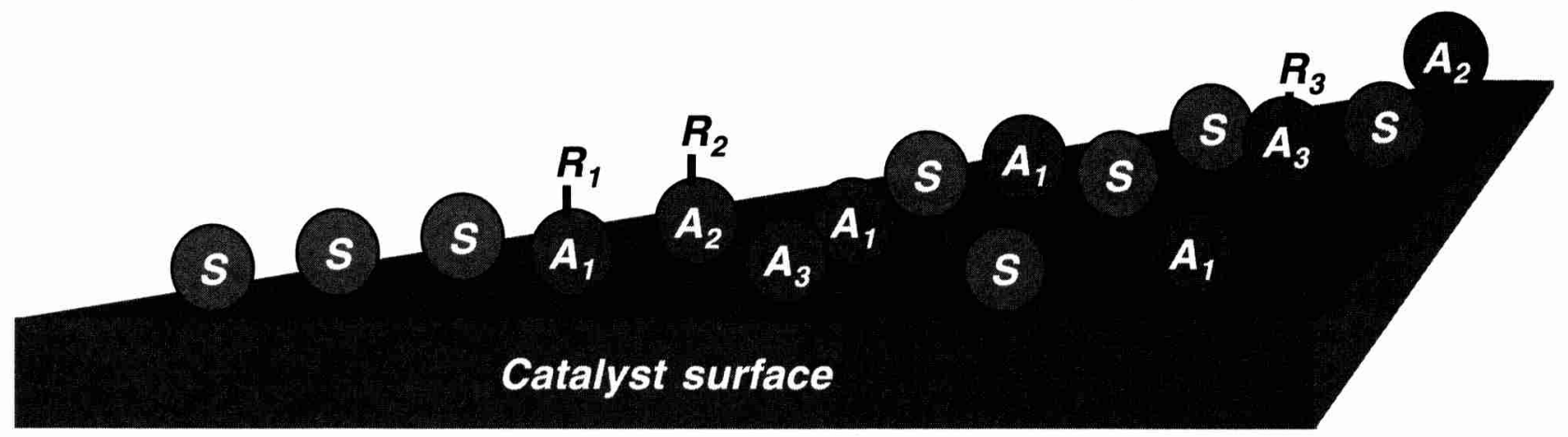

Figure 8. Complexity of catalytic solids, indicating the presence of different active sites $\left(A_{1}, A_{2}\right.$ and $\left.A_{3}\right)$, reaction intermediates $\left(R_{1}, R_{2}\right.$ and $\left.R_{3}\right)$ and spectator species (S). 
studies were mostly limited to the use of one particular in-situ spectroscopic technique measuring the catalyst under steadystate conditions, leading to spectroscopic data that did not change with increasing reaction time.

The disadvantages of this approach are threefold:

(1) The spectroscopic information obtained from a catalyst material under $e x$-situ conditions is of little relevance since important structural and physicochemical changes can take place in the catalyst material when present in the chemical reactor. The concessions made for reactor design and reaction temperature/pressure lead to information with sometimes little relevance for the working catalyst.

(2) Each spectroscopic technique has its own sensitivity and limitations (Table 3), meaning one technique will not provide all necessary chemical information (e.g. oxidation state, coordination environment, dispersion) about the working catalyst. Thus, more research has to be directed toward an intelligent combination of two or more spectroscopic techniques in order to provide complementary information on the molecular structure and composition of a catalyst under working conditions.

(3) The time resolution is often too low to detect reaction intermediates. One of the challenges is to discriminate between spectator species and species actively involved in the catalytic event, as well as to decrease the time necessary for measuring unusual states and reaction intermediates in an active catalyst. This requires the development of time-resolved techniques for measuring catalysts in the sub-second region in combination with fast on-line product analysis and transient kinetic experimentation.

Based on these observations, one can attempt a roadmap for in-situ spectroscopy and set the field for the years to come [11]. However, such an attempt risks failure or even incompletion. Nevertheless, it has the advantage that it may stimulate some discussion in the catalysis community. My ideas are summarized in Figure 9 and three domains of interest for future in-situ studies are proposed. Domain A can be named "measuring better, measuring faster and measuring more", whereas domain B concerns the logic extension of the operational window of in-situ studies to catalytic liquid-solid and liquid-gas-solid reaction systems. Domain $\mathrm{C}$ is much more speculative and can be named "single molecule and single site in-situ spectroscopymicroscopy". In what follows, I will discuss in detail how in-situ spectroscopy may develop in these three domains.

The first step in domain A, "measuring better", means that spectroscopic and catalytic measurements should be done on the same catalyst material at the same time and under optimal spectroscopic and catalytic conditions. Although it sounds simple, this is not often the case. The crucial step is to design the ideal in-situ cell in order to allow measuring at e.g. high

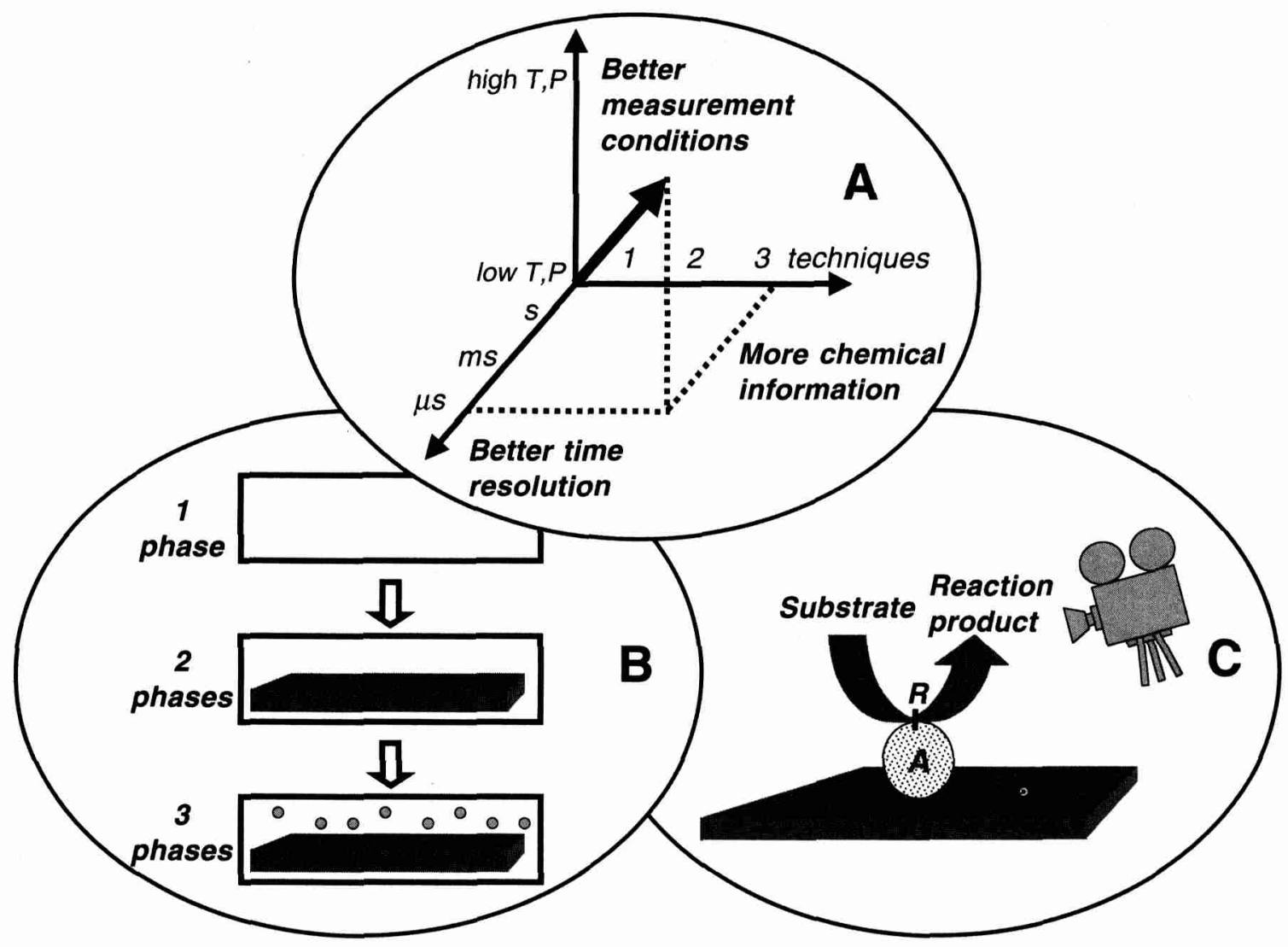

Figure 9. Potential roadmap for future in-situ spectroscopic studies. Three areas of interest are proposed: domain A, measuring better, measuring faster and measuring more; domain B, extension of the operational window from mono-phase over bi-phase to triphase reaction systems; domain $\mathrm{C}$, single molecule and single site in-situ spectroscopy-microscopy. 
temperatures and pressures. A detailed description on several aspects that must be taken into account when designing in-situ cells has been discussed before. Step 2 in domain A, "measuring faster", refers to developing the field of timeresolved spectroscopy in the sub-second region. This development should go hand in hand with the use of step/pulseresponse experiments to discriminate between active sites and spectator species. Finally, step 3 in domain A, "measuring more", implies that two or more spectroscopic techniques should be combined in one spectroscopic-reaction cell. This process introduces the multi-technique approach in the field of in-situ spectroscopy. Several studies in which different characterization techniques are combined have already appeared in literature: XRD-EXAFS [32-35], EPR-UV-VIS [36-37], EXAFS-UV-VIS [38] and UV-VIS-Raman [39].

Domain B concerns the expansion of the application domain of in-situ spectroscopy from mono-phase to multi-phase reaction systems. It is fair to say that in-situ spectroscopy of homogeneous catalysis (mono-phase reaction systems) is relatively simple because the catalyst material can be more easily explored with spectroscopic techniques. Within the field of heterogeneous catalysis (multi-phase reaction systems), one has to discriminate between bi-phase reaction systems (catalytic solid-gas and solid-liquid interfaces) and threephase reaction systems (catalytic gas-liquid-solid interfaces). Until now, most efforts in in-situ spectroscopy literature have been devoted to gas-solid reaction systems, whereas the study of catalytic solid-liquid and gas-liquid-solid reaction systems can be regarded as still in its infancy. There are, however, many challenges when studying the catalytic solid-liquid, and later, solid-liquid-gas interfaces. One of the most important difficulties is that the signals of interest from the interface are usually much smaller than the signals of the surrounding liquid phase and gas phase.

Domain $C$ is more speculative, but also very challenging. It could be named single molecule and single site chemistry as revealed by in-situ spectroscopy-microscopy. It would be wonderful if we could monitor the catalytic transformation of a substrate molecule on a particular active site and detect the formation of the corresponding reaction intermediately, and then detect that of the reaction product molecule. However, this would require focus on one single active site and the monitoring of the chemistry taking place at this site.

\section{CONCLUDING REMARKS}

Many catalyst discoveries are based on serendipity or on a trial-and-error approach often accelerated by the use of highthroughput experimentation (HTE) and design of experiment (DOE) techniques. Such discoveries continue to lead to new industrial catalytic processes. However, rational catalyst design should still be the ultimate goal of catalyst scientists simply because it is an intellectual challenge to design new catalysts based on fundamental knowledge about the active catalyst surface. To obtain such detailed information, researchers are developing and applying in-situ spectroscopic tools. The main challenges in this exciting research field are:

(a) The development of time-resolved spectroscopy, in conjunction with the design and construction of high quality spectroscopic-reaction cells, which allows the combination of complementary information of two or more characterization techniques.

(b) The study of catalytic solid-liquid and gas-liquid-solid reaction systems and the development of tools enabling the study of these catalytic interfaces.

(c) The combination of in-situ microscopy and spectroscopy with the possibility to focus on the catalytic chemistry of one single active site. This requires the development of site-selective spectroscopy-microscopy techniques.

In-situ spectroscopy is considered to be very important for the future development of catalysis science and engineering, and its developed methodologies will also be useful for gaining a better understanding of the fundamentals of catalyst synthesis.

\section{ACKNOWLEDGMENTS}

B. M. W. acknowledges the Dutch Science Foundation (NWO-CW) for a Van der Leeuw and VICI grant.

\section{REFERENCES}

1. Ertl, G., Knozinger, H., Weitkamp, J., (Eds.), Handbook of Heterogeneous Catalysis, Wiley-VCH, Weinheim, (1997).

2. Thomas, J. M., Thomas, W. J., Principles and Practice of Heterogeneous Catalysis, VCH, Weinheim, (1997).

3. Catalysis from A to Z, a concise encyclopedia, Cornils, B., Herrmann, W. A., Schlogl, R., Wong, C. H., (Eds.), Wiley-VCH, Weinheim, (2000).

4. Hagen, J., Industrial Catalysis, A practical approach, Wiley-VCH, Weinheim, (1999).

5. Rase, H. F., Handbook of Commercial Catalysts, CRC Press, New York, (2000).

6. Weckhuysen, B. M., Wachs, I. E., in Handbook of Surfaces and Interfaces of Materials, H.S. Nalwa (Ed.), Academic Press, San Diego, (2001), vol. 1, p. 613.

7. Niemantsverdriet, J. W., Spectroscopy in Catalysis, an introduction, VCH, Weinheim, (1993).

8. Weckhuysen, B. M., Van Der Voort, P., Catana, G., (Eds.), Spectroscopy of Transition Metal Ions on Surfaces, Leuven University Press, (2000).

9. Wachs, I. E., (Ed.), Characterization of Catalytic Materials, ButterworthHeineman, New York, (1992).

10. Weckhuysen, B. M., Chem. Comm., 97 (2002).

11. Weckhuysen, B. M., Phys. Chem. Chem. Phys., 5, 4351 (2003).

12. Hercules, D. M., Procter, A., Houalla, M., Acc. Chem. Res., 27, 387 (1994).

13. Jacoby, M., Chemical and Engineering News, May 4 1998, 41.

14. Thomas, J. M., Chem. Eur. J., 3, 1557 (1997).

15. Thomas, J. M., Angew. Chem. Int. Ed., 38, 3589 (1999).

16. Thomas, J. M., Catlow, C. R. A., Sankar, G., Chem. Comm., 2921 (2002).

17. Topsoe, H., Stud. Surf. Sci. Catal., 130, 1 (2000).

18. Topsoe, H., J. Catal., 216, 155 (2003).

19. Hunger, M., Weitkamp, J., Angew. Chem. Int. Ed., 40, 2954 (2001).

20. Grundwaldt, J. D., Wandeler, R., Baiker, A., Catal. Rev. Sci. Eng., 45, 1 (2003).

21. Bell, A. T., Stud. Surf. Sci. Catal., 92, 63 (1995).

22. Thomas, J. M., Somorjai, G. A., (Eds.), Top. Catal., 8, 1-140 (1999). Special issue with papers presented at the American Chemical Society National Meeting in Dallas (USA) in 1998. 
23. Schlogl, R., Zecchina, A., (Eds.), Top. Catal., 15, 1-82 (2001). Special issue with papers presented at the European Congress on Catalysis in Rimini (Italy).

24. Weckhuysen, B. M., (Ed.), Phys. Chem. Chem. Phys., 5, 4351-4515 (2003). Special issue with papers presented at the International Congress on Operando Spectroscopy. Operando spectroscopy is the shortened version of spectra of an operando or working catalyst.This name has been proposed by Miguel Banares, Eric Gaigneaux, Gerhard Mestl and Bert Weckhuysen at the 220th American Chemical Society National Meeting in Washington, D.C. (USA) in August 2000.

25. Haw, J. F., (Eds.), In-situ Spectroscopy in Heterogeneous Catalysis, Wiley-VCH, Weinheim, (2002).

26. Eischens, R. P., Plisken, W. A., Francis, S. A., J. Chem. Phys., 24, 1786 (1954).

27. Mapes, J. E., Eischens, R. P., J. Phys. Chem., 58, 1059 (1954).

28. Kurbatov, L. N., Nueimin, G. C., Doklady Akad. Nauk. S.S.S.R., 68, 341 (1949).

29. Yaroslavskii, N. G., Terenin, A. N., Doklady Akad. Nauk. S.S.S.R., 66, 885 (1949).
30. Yaroslavskii, N. G., Karayakin, A. V., Doklady Akad. Nauk. S.S.S.R., 85,1103 (1952).

31. This literature analysis is based on a Chemical Abstract search in the period 1967-2002 with the key-words catalyst/catalysis/catalytic and in-situ characterization/investigation. It is anticipated that not all papers on in-situ characterization of catalytic systems were found with this search and thus the given numbers have to be seen as estimations.

32. Clausen, B. S., Topsoe, H., Frahm, R., Adv. Catal., 42, 315 (1998).

33. Sankar, G., Thomas, J. M., Catlow, C. R. A., Top. Catal., 10, 255 (2000).

34. Sankar, G., Thomas, J. M., Top. Catal., 8, 1 (1999).

35. Grunwaldt, J. D., Clausen, B. S., Top. Catal., 18, 37 (2002).

36. Bruckner, A., Catal. Rev. Sci. Eng., 45, 97 (2003).

37. Bruckner, A., Chem. Commun., 2122 (2001).

38. Tromp, M., Sietsma, J. R. A., van Bokhoven, J. A., van Strijdonck, G. P. F., van Haaren, R. J., van der Eerden, A. M. J., van Leeuwen, P. W. N. M., Koningsberger, D. C., Chem. Commun., 128 (2003).

39. Nijhuis, T. A., Tinnemans, S. J., Visser, T., Weckhuysen, B. M., Phys. Chem. Chem. Phys., 5, 4361 (2003). 